Jenny Preunkert

Chancen für ein soziales Europa? 


\section{VS RESEARCH}

\section{Organization \& Public Management}

Herausgegeben von

Prof. Dr. Petra Hiller, Fachhochschule Nordhausen

Prof. Dr. Georg Krücken, Deutsche Hochschule für Verwaltungswissenschaften Speyer 
Jenny Preunkert

\section{Chancen für ein soziales Europa?}

Die Offene Methode der Koordinierung als neue Regulierungsform

Mit einem Geleitwort von Prof. Dr. Martin Heidenreich 
Bibliografische Information der Deutschen Nationalbibliothek

Die Deutsche Nationalbibliothek verzeichnet diese Publikation in der

Deutschen Nationalbibliografie; detaillierte bibliografische Daten sind im Internet über $<$ http://dnb.d-nb.de> abrufbar.

Dissertation Universität Oldenburg, 2009

1. Auflage 2009

Alle Rechte vorbehalten

(c) VS Verlag für Sozialwissenschaften | GWV Fachverlage GmbH, Wiesbaden 2009

Lektorat: Dorothee Koch / Anita Wilke

VS Verlag für Sozialwissenschaften ist Teil der Fachverlagsgruppe

Springer Science+Business Media.

www.vs-verlag.de

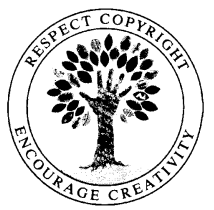

Das Werk einschließlich aller seiner Teile ist urheberrechtlich geschützt. Jede Verwertung außerhalb der engen Grenzen des Urheberrechtsgesetzes ist ohne Zustimmung des Verlags unzulässig und strafbar. Das gilt insbesondere für Vervielfältigungen, Übersetzungen, Mikroverfilmungen und die Einspeicherung und Verarbeitung in elektronischen Systemen.

Die Wiedergabe von Gebrauchsnamen, Handelsnamen, Warenbezeichnungen usw. in diesem Werk berechtigt auch ohne besondere Kennzeichnung nicht zu der Annahme, dass solche Namen im Sinne der Warenzeichen- und Markenschutz-Gesetzgebung als frei zu betrachten wären und daher von jedermann benutzt werden dürften.

Umschlaggestaltung: KünkelLopka Medienentwicklung, Heidelberg Gedruckt auf säurefreiem und chlorfrei gebleichtem Papier Printed in Germany

ISBN 978-3-531-16879-1 


\section{Geleitwort}

Die vorliegende Studie greift eine hochaktuelle Frage in der europawissenschaftlichen Debatte auf: Wie beeinflussen europäische Koordinierungsverfahren die Reformprozesse nationaler Sozialschutzsysteme? Am Beispiel dreier Länder, die bei der Reform ihrer wohlfahrtsstaatlichen Sicherungssysteme mit erheblichen Beharrungsmomenten konfrontiert sind (Deutschland, Frankreich und Italien), wird in der Arbeit diskutiert, ob ein europäisches, vor allem auf Freiwilligkeit und wechselseitigem Lernen beruhendes Koordinierungsverfahren, die Offene Methode der Koordinierung (OMK), die Nationalstaaten beim Kampf gegen soziale Ausgrenzung unterstützen kann. Die Arbeit stützt sich auf über 50 Interviews, die die Autorin von 2005-2007 in den drei genannten Ländern und auf der europäischen Ebene durchgeführt hat.

Auf europäischer Ebene werden die zentrale Stellung der Kommission als Katalysator des Prozesses und die nur begrenzten Lern- und Partizipationsmöglichkeiten für die Regierungen nachgewiesen. Auf nationaler Ebene zeigt die Studie, dass der Einfluss des europäischen Verfahrens nur verstanden werden kann, wenn die nationalen Rahmenbedingungen bekannt sind. Angesichts der Zersplitterung sozialpolitischer Kompetenzen in Deutschland kommt europäischen Zielen und Indikatoren nur eine geringe Bedeutung für nationale Reformprozesse $\mathrm{zu}$ - auch wenn die Wohlfahrtsverbände erheblich von den neuen Lernund Partizipationschancen profitieren. Ganz anders ist die Situation in Frankreich, wo die nationalen Entscheidungsträger in der Verwaltung auch in die europäischen Koordinierungsprozesse einbezogen werden und damit die Wirksamkeit des Verfahrens institutionell sicherstellen. Paradoxerweise scheinen gerade die zentralistisch-expertokratischen Strukturen der französischen Sozialpolitik eine wichtige Voraussetzung für neue Partizipations- und Lernchancen zu sein. Der italienische Fall hingegen zeigt, dass ohne einen politischen Willen zur Reform, ohne einen handlungsfähigen Staat und ohne die Bereitschaft zur zentralen Moderation sozialpolitischer Reformprozesse die OMK kaum wirksam ist. Im Gegensatz zu vielen Arbeiten, die euphorisch die mit der OMK verbundenen Lern- und Partizipationsmöglichkeiten hervorheben, folgert die Verfasserin, dass die OMK/Inklusion „keinen Beitrag dazu leisten konnte, den nationalen Kampf gegen soziale Ausgrenzung umfassend und nachhaltig zu stärken und auszubau- 
en.“ (S. 352) Vielmehr nutzen die Regierungen die europäischen Koordinierungsprozesse jeweils im Rahmen der eigenen Problemwahrnehmungen, Reformstrategien und Verwaltungs- und Entscheidungsstrukturen. Die Arbeit von Frau Dr. Preunkert leistet somit einen sowohl in theoretischer wie auch in empirischer Hinsicht überzeugenden Beitrag zur Debatte um die Wirksamkeit weicher europäischer Regulierungsinstrumente. Sie belegt auf beeindruckende Weise, dass die untersuchten Koordinierungsverfahren ihre Wirkung nur unter Berücksichtigung der nationalen Rahmenbedingungen, Strukturen und Interessen entfalten kann. Daher wünsche ich dieser Arbeit eine breite Rezeption.

Prof. Dr. Martin Heidenreich Jean Monnet Centre for Europeanisation and Transnational Regulations, Oldenburg 


\section{Inhaltsverzeichnis}

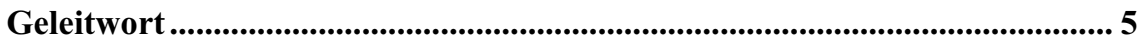

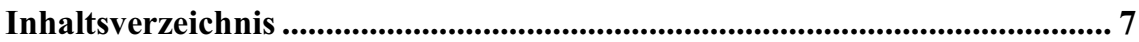

Abbildungsverzeichnis .................................................................................................... 13

Abkürzungsverzeichnis .................................................................................................. 15

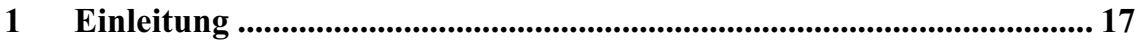

2 Debatten um die Offenen Methode der Koordinierung im Bereich soziale Eingliederung ................................................................................... 27

2.1 Rahmenbedingungen einer europäischen Sozialpolitik ...................... 28

2.2 Die Offene Methode der Koordinierung als Regulierungsverfahren .... 36

2.2.1 Die organisatorischen Besonderheiten der Offenen Methode....... 37

2.2.2 Die Lernprozesse im Rahmen weicher Regulierungsverfahren .... 41

2.2.3 Auswirkungsmöglichkeiten der OMK-Prozesse ........................... 45

2.3 Schlussfolgerungen und offene Fragen.............................................. 50

3 Die Offene Methode der Koordinierung: Die wechselseitigen

Irritationen von nationalen und europäischen Feldern ........................... 53

3.1 Kennzeichen eines sozialen Feldes ................................................... 55

3.2 Entstehung eines europäischen Raums ..............................................6 60

3.2.1 Die soziale Konstruktion eines europäischen Feldes .................... 61

3.2.2 Die Kopplungsprozesse zwischen europäischen und nationalen

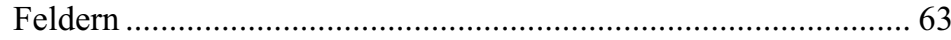

3.2.3 Zwischenresümee: Voraussetzungen für die Wirksamkeit

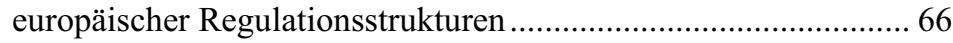

3.3 Die nationale Implementierung europäischer Impulse ....................... 67

3.3.1 Exkurs: Das Prinzip der Pfadabhängigkeit ..................................... 68

3.3.2 Individuelles, organisationales und institutionelles Lernen .......... 69

3.3.3 Der europäische Prozess als Anreiz und Ressource...................... 72

3.3.4 Zwischenresümee: Mögliche Auswirkungen von europäischen

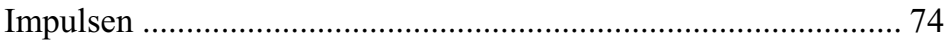

3.4 Schlussfolgerungen und Hypothesen ................................................. 75 


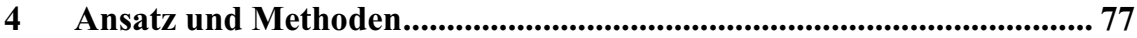

4.1 Methodenprobleme der OMK-Forschung........................................... 77

4.2 Qualitativ-komparative Fallstudien .................................................... 80

4.3 Länderauswahl und zeitliche Einschränkungen ................................... 81

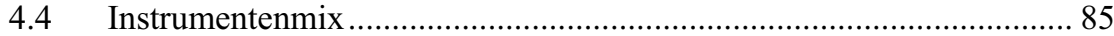

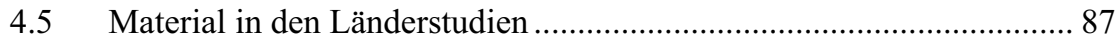

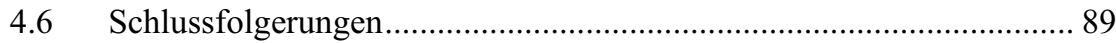

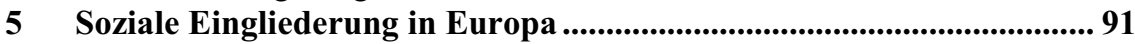

5.1 Der historische Kontext der OMK/Inklusion..................................... 92

5.1.1 Aktionsprogramme in den 1970er bis 1990er Jahren................... 93

5.1.2 Diskussionen auf europäischer Ebene.......................................... 95

5.1.3 Zwischenresümee: Von der Armutsbekämpfung hin zum Kampf gegen soziale Ausgrenzung............................................. 98

5.2 Die Organisation des europäischen Felds ......................................... 100

5.2.1 Die rechtliche Verankerung der OMK/Inklusion in den Verträgen von Amsterdam und Nizza......................................... 102

5.2.2 Das Zentrum der OMK/Inklusion: Der Sozialschutzausschuss .. 104

5.2.3 Begrenztes Interesse: Der Ausschuss des Aktionsprogramms.... 109

5.2.4 Koordinator und Stratege: Die Kommission................................ 109

5.2.5 Impulsgeber: Die Rolle der Ratspräsidentschaft........................ 113

5.2.6 Hierarchische Partizipation: Einbindung von nichtstaatlichen, wissenschaftlichen Organisationen und dem Europäischen

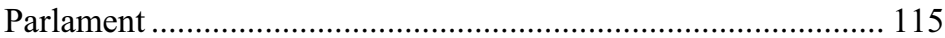

5.2.7 Zwischenresümee: Interaktionsmuster....................................... 122

5.3 Die Instrumente der OMK/Inklusion ................................................ 124

5.3.1 Vorbehalte gegen quantifizierbare Ziele: Die Erstellung gemeinsamer Ziele .................................................................. 125

5.3.2 Wissenschaftlich robuste Indikatoren ohne ein Ranking der Politikansätze .......................................................................... 129

5.3.3 Abstraktes Kennenlernen ohne substanzielle Analyse: Die PeerReview-Verfahren................................................................. 132

5.3.4 Mehr als der kleinster Nenner, aber nur bedingt kritisch: Die Gemeinsamen Berichte ............................................................. 133

5.3.5 Problembezogenes Lernen und Aufbau von transnationalen nichtstaatlichen Beziehungen: Das Aktionsprogramm ................ 137

5.3.6 Zwischenresümee: Abstrakte und konkrete Lernchancen............ 142

$5.4 \quad$ Die Leitbilder der OMK/Inklusion ...................................................... 143

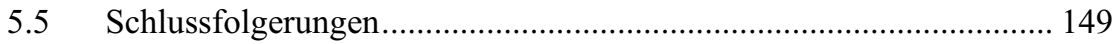


6 Die Offene Methode der Koordinierung in Deutschland ...................... 153

6.1 Soziale Eingliederung in Deutschland ............................................... 154

6.1.1 Armut als bekämpftes Phänomen und die Aktivierung von Leistungsempfängern................................................................ 155

6.1.2 Der Armuts- und Reichtumsbericht ............................................ 160

6.1.3 Breite Kompetenzverteilung .................................................... 162

6.1.4 Zwischenresümee: Zwischen Wandel und nicht-hinterfragten

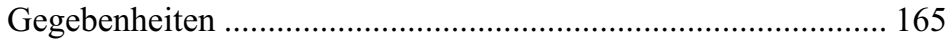

6.2 Die Organisation der Offenen Methode der Koordinierung ............... 166

6.2.1 Skeptisches Koordinationszentrum: Die Bundesregierung ......... 167

6.2.2 Föderale Machtkämpfe: Die Einbindung der Bundesländer ....... 172

6.2.3 Ausgeschlossen: Die Kommunen ........................................... 174

6.2.4 Aktiv handelnde und beratende Organisationen: Die

6.2.5 Zwischenresümee: Die OMK/Inklusion als Nebensache............ 178

6.3 Die Umsetzung des europäischen Verfahrens ................................... 179

6.3.1 Unpassende Vorgaben: Die Ziele und Indikatoren der OMK/Inklusion ..................................................................... 180

6.3.2 Die Umsetzung der Nationalen Aktionspläne als europäische

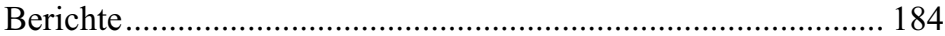

6.3.3 Selbstdarstellung ohne Folgen: Die Beteiligung an den Gemeinsamen Berichten mit den bewährten Praxisbeispielen und den Peer-Review-Verfahren................................................ 188

6.3.4 Punktuelles Lernen in den staatlichen Stellen, breitere Nutzung durch die Zivilgesellschaft: Das Aktionsprogramm..... 191

6.3.5 Zwischenresümee: Punktuelle Impulse......................................... 197

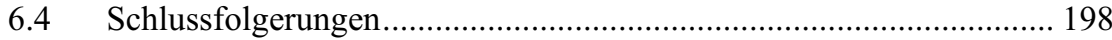

7 Die Offene Methode der Koordinierung in Frankreich ....................... 201

7.1 Soziale Eingliederung in Frankreich................................................ 202

7.1.1 Das Konzept der sozialen Kohäsion .......................................... 203

7.1.2 Eine Politik der sozialen Kohäsion .............................................. 204

7.1.3 Ausbau von staatlichen Maßnahmen .......................................... 208

7.1.4 Ein neues Budgetrecht .......................................................... 212

7.1.5 Zentralistische Kompetenzverteilung.......................................... 213

7.1.6 Zwischenresümee: Feld zur Stärkung der sozialen Kohäsion..... 218

7.2 Die Organisation der Offenen Methode der Koordinierung ............... 219

7.2.1 Die Rolle der Regierung und ihrer nationalen Verwaltung......... 219

7.2.2 Die Informierung der übrigen Organisationen ............................ 223

7.2.3 Zwischenresümee: OMK/Inklusion als administrativer Prozess. 226 
7.3 Die Umsetzung des europäischen Verfahrens ................................. 227

7.3.1 Politisches Präsentieren und administratives Lernen: Die

Nutzung der Ziele und Benchmarkingverfahren ........................ 228

7.3.2 Argumente und Lernhilfe für das neue Budgetrecht: Die

Nutzung der Indikatoren

7.3.3 Stärkung der interministeriellen Zusammenarbeit und Erarbeitung einer gemeinsamen Strategie: Die Erstellung der Aktionspläne .....

7.3.4 Generelles Kennenlernen und Anregungen für weitere Lernprozesse: Die Teilnahme an den Peer-Reviews

7.3.5 Lernmöglichkeit für die Administration und einzelne Mitarbeiter des nichtstaatlichen Sektors:

Das Aktionsprogramm

7.3.6 Zwischenresümee: Administrative Nutzung bei fehlendem politischen wie nichtstaatlichen Interesse .................................. 242

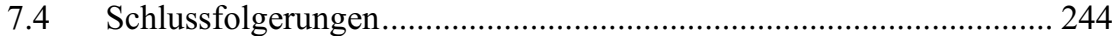

8 Die Offene Methode der Koordinierung in Italien............................... 247

8.1 Soziale Eingliederung in Italien..................................................... 248

8.1.1 Die patriarchale Familie als Leitbild........................................ 249

8.1.2 Erster Wendepunkt: Das Gesetz 328/2000 im Jahr 2000............ 253

8.1.3 Der zweite Wendepunkt: Das Weißbuch im Jahr 2003 .............. 255

8.1.4 Dezentrale Kompetenzverteilung............................................... 258

8.1.5 Zwischenresümee: Ein fragmentiertes Feld ............................... 262

8.2 Die Organisation der Offenen Methode der Koordinierung ............... 263

8.2.1 Schwaches Koordinationszentrum: Die Regierung..................... 264

8.2.2 Die geringe Einbindung der lokalen Ebenen ............................. 267

8.2.3 Unabhängig von der Regierung: Der nichtstaatliche Sektor ....... 269

8.2.4 Zwischenresümee: Fehlendes staatliches Interesse.................... 270

8.3 Die Umsetzung des europäischen Verfahrens ................................. 271

8.3.1 Strategische, einseitige Nutzung und fehlende Akzeptanz: Die Ziele und Indikatoren der OMK/Inklusion ................................ 272

8.3.2 Fehlendes Konzept und mangelndes Interesse: Die Nutzung der Aktionspläne .................................................................... 275

8.3.3 Weder gegenseitiges Lernen noch informeller Handlungsdruck: Benchmarking auf europäischer Ebene .......... 279

8.3.4 Nutzung des Aktionsprogramms durch den nichtstaatlichen Sektor

8.3.5 Zwischenresümee: Zwischen Legitimation und tatsächlichem

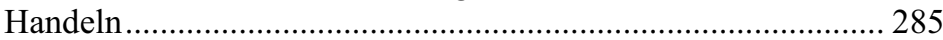

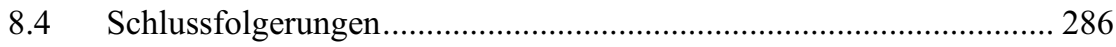


9 Die Neuausrichtung der Offenen Methode der Koordinierung.......... 289

9.1 Synchronisierung und Straffung ..................................................... 289

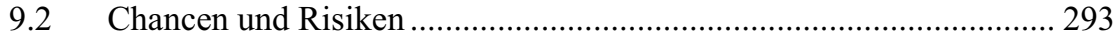

9.3 Kritische Prognose: Tendenz zur Verallgemeinerung und Ausrichtung auf wirtschaftliche Interessen........................................ 297

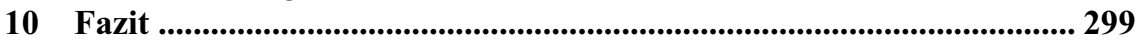

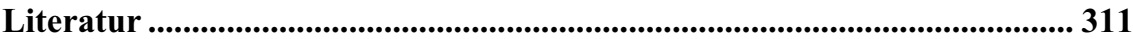

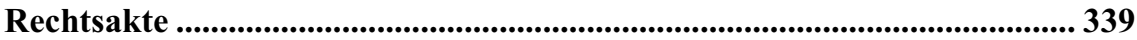




\section{Abbildungsverzeichnis}

Abbildung 1: Mechanismen der Kopplung zwischen sozialen Feldern ........... 66

Abbildung 2: Ebenen, die beeinflusst werden können .................................... 71

Abbildung 3: Europäische Impulse in ein nationales Feld .............................. 73

Abbildung 4: Auswahl der Gesprächspartner................................................ 90

Abbildung 5: Armutsrisiko für einzelne Bevölkerungsgruppen in

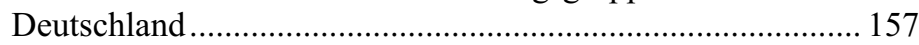

Abbildung 6: Formen der sozialen Hilfe nach den Hartz-IV-Reformen ........ 159

Abbildung 7: Erstellung der Nationalen Aktionspläne in Deutschland.......... 185

Abbildung 8: Armutsrisiko für einzelne Bevölkerungsgruppen in

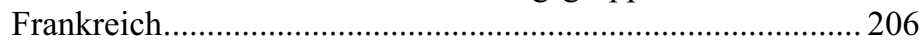

Abbildung 9: Erstellung der Nationalen Aktionspläne in Frankreich ............. 235

Abbildung 10: Armutsrisiko für einzelne Bevölkerungsgruppen in Italien ..... 251

Abbildung 11: Zentrale Punkte des Weißbuches ........................................... 256

Abbildung 12: Erstellung der Nationalen Aktionspläne in Italien ................... 278 


\section{Abkürzungsverzeichnis}

$\begin{array}{ll}\text { ALG II } & \text { Arbeitslosengeld II } \\ \text { ARGE } & \text { Arbeitsgemeinschaften } \\ \text { ASMK } & \text { Arbeits- und Sozialministerkonferenz } \\ \text { ASS } & \text { Allocation speciale que de solidarité } \\ \text { AStV } & \text { Ausschuss der Ständigen Vertreter } \\ \text { ATD } & \text { All Together for Dignity } \\ \text { AWO } & \text { Arbeiterwohlfahrt } \\ \text { BAEI } & \text { Bureau des affaires européennes et internationales } \\ \text { BAGFW } & \text { Bundesarbeitsgemeinschaft der freien Wohlfahrtspflege e.V. } \\ \text { BMAS } & \text { Bundesministeriums für Arbeit und Soziales } \\ \text { BMFSFJ } & \text { Bundesministerium Familien, Senioren, Frauen und Jugend } \\ \text { BMGS } & \text { Bundesministerium für Gesundheit und Soziale Sicherung } \\ \text { BMWA } & \text { Bundesministerium für Wirtschaft und Arbeit } \\ \text { CES } & \text { Conseil économique et social } \\ \text { CIG } & \text { Cassa Integrazione Guadagni } \\ \text { CILAP } & \text { Collegamento Italiano di Lotta alla Povertà } \\ \text { CILE } & \text { Comité interministériel de lutte contre des exclusions } \\ \text { CNLE } & \text { Conseil nationale des politique de lutte contre la pauvreté et } \\ & \text { l'exclusion sociale } \\ \text { DGAS } & \text { Direction générale de l'action sociale } \\ \text { DGEFP } & \text { Délégation générale à l'emploi et à la formation profession- } \\ & \text { nelle } \\ \text { DPT } & \text { Document de politique transversale } \\ \text { DREES } & \text { Direction de la recherche, des études, de l'évaluation et, des } \\ & \text { statistiques } \\ \text { EAPN } & \text { European Anti-Poverty Network } \\ \text { EBS } & \text { Europäische Beschäftigungsstrategie } \\ \text { EP } & \text { Europäisches Parlament } \\ \text { E-Referat } & \text { Europareferat } \\ \text { ESF } & \text { Europäischer Sozialfond } \\ \text { ESN } & \text { Europäischen Sozialen Netzwerk } \\ \text { EWSA } & \text { Europäischer Wirtschafts- und Sozialausschuss } \\ & \end{array}$


FEANTSA European Federation of National Organisations working with the Homeless

FORTEIL Forum Teilhabe und soziale Integration

GD Generaldirektion

INPS Istituto Nazionale della Previdenza Sociale

KOM Europäische Kommission

LOLF Loi Organique Relative aux Lois de Finances

NAK Nationalen Armutskonferenz

NAP/Inklusion Nationale Aktionspläne Soziale Eingliederung

NAPsens Nationale Sensibilisierungsmaßnahmen zum Thema, Soziale Integration'

NGO Nichtregierungsorganisation

OMK Offene Methode der Koordinierung

OMK/Inklusion Offene Methode der Koordinierung Soziale Eingliederung

PROGRESS Gemeinschaftsprogramms für Beschäftigung und soziale Solidarität

RAT Rat der Europäischen Union

RETIS European Transregional Network for Social Inclusion

RMI Revenu Minimum d'Inseration

RUI Reddito di Ultima Istanza

SGAE Secrétariat général des affaires européennes

SPC

UNEDIC

Ausschuss für Sozialschutz oder Sozialschutzkomitee

Union national pour l'emploi dans l'industrie et le commerce Arbeitslosenversicherungsverwaltung

UNIOPSS Union nationale interfédérale des œuvres et organismes privés sanitaires et sociaux 